Cita: Rodrigues, F., Macedo, R., Teixeira, D., Cid, L., \& Monteiro, D. (2021). Análise comportamental da prática de exercício físico em adultos em contexto de ginásio ao longo de dois anos. Cuadernos de Psicología del Deporte, 21(1), 282-292

\title{
Análise comportamental da prática de exercício físico em adultos em contexto de ginásio ao longo de dois anos
}

\section{Análisis del comportamiento del ejercicio físico en adultos en el gimnasio durante dos años}

\section{Analysis of the exercise behavior in gym exercisers across two years}

\author{
Filipe Rodrigues ${ }^{1,2,3}$, Rita Macedo ${ }^{4}$, Diogo Teixeira ${ }^{5,6}$, Luís Cid ${ }^{1,3}$, Diogo Monteiro ${ }^{7,3}$ \\ 1 Escola Superior de Desporto de Rio Maior (ESDRM-IPSantarém), Rio Maior, Portugal; 2 Centro de \\ Investigação em Qualidade de Vida (CIEQV), Rio Maior, 3 Centro de Investigação em Desporto, Saúde e \\ Desenvolvimento Humano (CIDESD), Vila Real, Portugal; 4 Centro de Estudos Organizacionais e \\ Sociais do Politécnico do Porto (CEOS - PP), Porto, Portugal; 5 Universidade Lusófona de \\ Humanidades e Tecnologias, Faculdade de Educação Física e Desporto (ULHT/FEFD); 6 Centro \\ Interdisciplinar de Estudo da Performance Humana (CIPER), Lisboa, Portugal; 7 ESECS, Politécnico de \\ Leiria, Leiria, Portugal.
}

\begin{abstract}
RESUMO
O objetivo deste estudo consistiu em analisar o comportamento dos praticantes de exercício físico ao longo de dois anos, considerando a sua frequência como preditor da frequência futura. Participaram neste estudo 4788 novos praticantes de exercício físico (feminino $=2556$ ) com idades compreendidas entre os 18 e 75 anos. Os acessos aos ginásios e health clubs foi recolhida com recurso aos sistemas eletrónicos dos ginásios. Os dados foram analisados com o recurso a um modelo de equações estruturais e um modelo de mediação em série. Relativamente à taxa de abandono e posteriormente exclusão da análise: $39 \%$ abandonaram a prática de exercício físico entre os primeiros seis meses (T1) e os seis meses seguintes (T2); 25\% desistiu da prática entre T2 e os seis meses após um ano de prática regular (T3); e 10\% abandonaram entre T3 e os seis meses após um ano e meio de prática regular (T4). De acordo com os resultados do modelo de equações estruturais, foram encontrados diversos efeitos diretos e indiretos significativos, sugerindo assim que T2 e T3 poderão ser mediadores entre o T1 e T4. De acordo com os resultados do modelo de mediação é possível observar que o efeito direto não é significativo. Os resultados mostram a existência de mediação total entre o período $\mathrm{T} 1 \rightarrow \mathrm{T} 4$, dado o efeito indireto total ser significativo e superior ao efeito direto. Os indivíduos que praticam exercício físico pelo menos duas vezes por semana sem interrupção foram aqueles que demonstraram maior probabilidades em se manterem na prática após os dois anos de acompanhamento do comportamento.
\end{abstract}

Palavras chave: exercício; adesão; persistência; análise longitudinal; mediação. 


\title{
Rodrigues, F., Macedo, R., Teixeira, D., Cid, L., \& Monteiro, D.
}

\begin{abstract}
This study aimed to examine the behavior of exercisers across two years, considering their exercise frequency as a predictor of future frequency. In total, 4788 new gym exercisers (female $=2556$ ) aged between 18 and 75 years were enrolled for analysis. Adherence. Computerized records at the gyms and health clubs were used to measure exercise adherence. The collected data was analyzed using structural equation modelling and mediation model specifications. Regarding the dropout rate and later exclusion from the analysis: 39\% dropped out within the first six months (T1) and the following six months (T2); $25 \%$ withdraw the practice between T2 and six months after one year of regular practice (T3); and 10\% dropped out between T3 and six months after a year and a half of regular practice (T4). According to the results of the structural equation model, several significant direct and indirect effects were found, thus suggesting that T2 and T3 may play a mediation role between T1 and T4. Looking at the results from the mediation model, it is possible to observe that the direct effect was not significant. The results show the existence of total mediation between the period $\mathrm{T} 1 \rightarrow \mathrm{T} 4$, since the total indirect effect is significant and greater than the direct effect. Individuals who practice physical exercise at least twice a week without interruption were those who were more likely to remain in practice after two years of monitoring their behavior.
\end{abstract}

Keywords: exercise; adherence, persistence, longitudinal analysis; mediation.

\section{RESUMEN}

El objetivo de este estudio fue analizar el comportamiento de los practicantes de ejercicio físico durante dos años, considerando su frecuencia como un predictor de la frecuencia futura. Participaron en este estudio 4788 nuevos practicantes de ejercicio físico (mujeres $=2556$ ) con edades comprendidas entre 18 y 75 años. El acceso a gimnasios y health clubs se recopiló utilizando los sistemas electrónicos. Los datos se analizaron utilizando un modelo de ecuación estructural y un modelo de mediación. En cuanto a la tasa de abandono y posterior exclusión del análisis: $39 \%$ abandonó la práctica de ejercicio físico entre los primeros seis meses (T1) y los siguientes seis meses (T2); 25\% abandonó la práctica entre T2 y seis meses después de un año de práctica regular (T3); y 10\% abandonó entre T3 y seis meses después de un año y medio de práctica regular (T4). Según los resultados del modelo de ecuaciones estructurales, se encontraron varios efectos directos e indirectos significativos, lo que sugiere que T2 y T3 pueden ser mediadores entre T1 y T4. Según los resultados del modelo de mediación, es posible observar que el efecto directo no es significativo. Los resultados muestran la existencia de una mediación total entre el período T1 $\rightarrow$ T4, ya que el efecto indirecto total es significativo y superior al efecto directo. Las personas que practican ejercicio físico al menos dos veces por semana sin interrupción fueron las que tenían más probabilidades de permanecer en la práctica después de dos años de monitorear su comportamiento.

Palabras llave: ejercicio; adhesión; persistencia; análisis longitudinal; mediación.

\section{INTRODUÇÃO}

A falta da prática de exercício físico regular constitui-se ainda, nos dias de hoje, um problema de saúde pública. Embora exista profusa literatura dos benefícios associados à prática regular de atividade física (Janssen \& LeBlanc, 2010), 68\% da população portuguesa é fisicamente inativa, taxa esta que tem vindo a aumentar ao longo dos últimos anos (Eurobarómetro, 2018). Consequentemente, o número de pessoas portadoras de doenças crónicas não transmissíveis (e.g., hipercolesterolemia, enfarte do miocárdio, obesidade) tem aumentado, afetando os sistemas nacionais de saúde com despesas públicas amplificadas (Organização Mundial de Saúde - OMS, 2018), assim como a qualidade de vida e longevidade da população mundial (Arem et al., 2015).

Como forma de combater esta tendência, muito foco se tem dado à prática de exercício físico em instalações apropriadas e supervisionadas por profissionais, tais como em ginásios e health clubs (Teixeira et al., 2020). O exercício físico define-se 


\section{Prática de exercício físico em adultos em contexto de ginásio}

como a prática regular e estrutura de atividade física (Caspersen et al., 1985). Assim sendo, assume-se que quem pratica exercício físico, que $o$ faça semanalmente de forma regular, dentro dos padrões estruturados tais como aulas de grupo ou treino de força e resistência. Em 2018, registaram-se aproximadamente 539.000 praticantes inscritos, sendo que destes, 340.000 eram novos sócios (Associação Empresarial de Ginásios e Academias em Portugal AGAP, 2018). Ou seja, assume-se que $37 \%$ dos praticantes inscritos no ano anterior se mantiveram na prática, mostrando que a taxa de abandono possa ter atingido aproximadamente $63 \%$. Estes números demonstram bem a elevada taxa de desistência da prática de exercício físico em ginásios e health clubs e como o estudo do comportamento do praticante se torna uma necessidade essencial (Rodrigues et al., 2018; 2020a). Contudo, é de salvaguardar que não existem estudos que indiquem a transição da prática de exercício físico de forma estruturada em ginásios para uma vertente mais autodidata tais como caminhadas e ou programas de atividade física comunitários.

Olhando para a perspetiva individual, aproximadamente $50 \%$ dos novos praticantes de exercício físico abandonam o comportamento nos primeiros 6 meses (Radel et al., 2017). De acordo com Gardner (2015), a introdução de um comportamento novo no quotidiano de uma pessoa constitui-se como um processo complexo que necessita de tempo até se tornar habitual. Hagger (2018) reforça esta afirmação, constatando o facto de que a formação do comportamento habitual ou rotineiro é fortalecida pela realização repetida desse mesmo comportamento. Por outras palavras, quanto maior for a frequência (a repetição) do comportamento, maior será a probabilidade de esse comportamento vir a ser reiterado no futuro. Neste sentido, a execução de um comportamento habitual requer menos esforço e menos atenção consciente comparativamente a um comportamento não habitual (Brickell et al., 2006). De acordo com Barg e Ferguson (2000), um comportamento habitual parece estar sob o controle inconsciente da pessoa, sendo assim a sua realização um processo repetido e facilitado.

A literatura é consensual no que diz respeito ao impacto que o comportamento passado tem no comportamento futuro (Sommers, 2011) inclusive em estudos empíricos no contexto do exercício físico (Rodrigues et al., 2019a; Rodrigues et al., 2020b). Ou seja, a repetição de um comportamento pode vir a tornar-se habitual, fazendo parte do registo quotidiano de uma pessoa, caso este comportamento seja repetido ao longo do tempo. Tomando em consideração o exemplo de subir um lanço de escadas, indivíduos que usaram no passado regularmente o elevador para chegar de um piso ao outro, estão mais predispostos a repetirem o comportamento num próximo momento. Igualmente, pessoas que usam as escadas optam por manter esse comportamento no futuro. Não obstante, pessoas que usam frequentemente o elevador estão menos propensas a usarem as escadas e vice-versa. Este exemplo tem vindo a ser usado por investigadores para mostrar a automaticidade do comportamento e como a repetição de um comportamento específico reforça a sua repetição, dificultando o processo de mudança para outro comportamento (Nomura et al., 2009). Adicionalmente, parece inegável que a natureza da experiência passada (de natureza subjetiva), seja de particular relevo para a definição da sua relação com o comportamento futuro, motivo pelo qual se eleva a necessidade de os profissionais de exercício físico estarem devidamente preparados para lidar com os clientes nas diferentes fases desta mudança comportamental (Rodrigues et al., 2019b; Teixeira et al., 2020).

Considerando os pressupostos teóricos anteriores, o estudo do comportamento humano e o seu valor preditor é uma área de investigação decisiva (Gardner, 2015). Embora a literatura afirme consistentemente que o comportamento realizado no passado prediz fortemente a sua realização no futuro, este tipo de análise no contexto do exercício físico é escasso (Rodrigues et al., 2020b). Investigadores têmse focado na análise de variáveis preditoras nãoobserváveis (e.g., motivação, bem-estar) colocando de lado o estudo do comportamento mensurável per se. Estudos anteriores têm demonstrado que determinantes cognitivas (Gomes et al., 2017) e motivacionais (Hagger \& Chatzisarantis, 2017) pouco poder explicativo têm na formação do comportamento futuro. Aliás, os dados recolhidos através de questionários autoadministrados relativamente à adesão e frequência ao exercício físico parecem apresentar viés nos resultados (Liu et al., 2016). Tal premissa, parece estar relacionada com o facto de as pessoas não terem a certeza da quantidade ou se terem esquecido das vezes que realizaram o comportamento no passado, como por exemplo as vezes que treinaram 


\section{Rodrigues, F., Macedo, R., Teixeira, D., Cid, L., \& Monteiro, D.}

no ginásio ou health club (Brennder \& DeLamater, 2016).

No entanto, a investigação que estuda a frequência da prática de exercício físico e a sua associação com o comportamento é, ainda, escassa e os resultados dos estudos revelam alguma inconsistência (Rodrigues et al., 2020a). Por um lado, o estudo de Rodrigues et al. (2020b) demonstrou que o comportamento passado é o preditor mais significativo do comportamento futuro. Por outro lado, no estudo de Gardner (2015) uma maior experiência na realização do comportamento não estava significativamente associada à formação do hábito e na realização do comportamento. Adicionalmente, pretende-se explorar as limitações propostas por Teixeira et al. (2020) no que diz respeito à falta de dados e estudos que quantifiquem adequadamente a prática de exercício físico a longo prazo em ginásios e health clubs, de forma a os profissionais possam estar munidos do panorama atual da prática de exercício físico em operadores fitness. Os resultados poderão dar a conhecer a realidade nacional no que diz respeito à frequência por parte dos utilizares, permitindo, por exemplo, uma aferição externa da qualidade das práticas por parte dos profissionais para que os operadores de fitness possam ser reconhecidos como locais adequados na promoção da saúde (Teixeira et al., 2020).

Assim, o objetivo deste estudo consistiu em analisar comportamento dos praticantes de exercício físico em ginásios e health clubs ao longo de dois anos, considerando a frequência passada como preditor da frequência futura. De acordo com estudos prévios, especula-se que: a) os primeiros 6 meses são um período "crítico", em que a taxa de abandono poderá rondar os 50\% (Radel et al., 2017); b) praticantes com mais de 1 ano de experiência e comportamento regular mantém-se na prática, sendo que, o valor preditivo seja mediado pelo comportamento passado (Rodrigues et al., 2020b; Verplanken, 2006); e c) a frequência (i.e., entradas no ginásio ou health club) regular sem episódios de desistência prediz a manutenção do comportamento no futuro (Lally et al., 2011).

\section{MÉTODOS}

\section{Participantes}

Participaram neste estudo 4788 indivíduos $($ feminino $=2556 ;$ masculino $=2232)$ com idades compreendidas entre 18 e 45 anos $(\mathrm{M}=25.21$; $\mathrm{DP}=$ 5.23). Estes indivíduos eram praticantes de exercício físico em ginásios e health clubs em Portugal em diversas atividades fitness, tais como aulas de grupo, personal training e musculação. A amostra era principalmente constituída por trabalhadores (65\%), sendo a restante amostra constituída por estudantes $(12 \%)$ e por indivíduos reformados $(23 \%)$. A amostra é relativa de ginásios e health clubs do território português, sendo a maioria da amostra residente em grandes cidades tais como Lisboa (30\%), Porto (28\%) e Braga $(21 \%)$.

Como critérios de inclusão, os participantes tinham de ter idade igual ou superior a 18 anos, serem novos clientes no ginásio e terem todas as quotas mensais pagas durante o período em análise. Foram excluídos da análise todos os indivíduos que tivessem abandonado a prática e/ou que não tivessem retomado à prática, dentro do período de 2 anos estabelecidos neste estudo.

\section{Procedimentos}

Após aprovação pela Comissão de Ética ( $n^{\circ}$ de registo CE-UBI-pJ-2018-044:ID683), foram contactados por conveniência gestores de ginásios e health clubs. Os objetivos do estudo foram explicados e o consentimento foi obtido de todos os gestores de ginásios e health clubs para a recolha dos dados. Foram considerados os registos eletrónicos dos clientes de ginásio para fins desta investigação. Este estudo é considerado um estudo de coorte prospetivo. Assim sendo, este estudo é observacional na sua natureza, ao qual não houve intervenção nem randomização da amostra em grupos. Os investigadores recolheram os dados de acesso ao ginásio e health club, bem como variáveis para a caracterização da amostra tais como idade e sexo. Nenhum cliente de ginásio e/ou health club foi abordado, dado que os dados terem sido recolhidos através dos sistemas eletrónicos. Foi garantida a confidencialidade e anonimato a todos os gestores de ginásios e health clubs.

Os investigadores iniciaram a recolha de dados a setembro de 2017 e terminaram em janeiro de 2020 . Foi recolhida a informação dos acessos ao ginásio e health clubs dos novos praticantes de exercício durante 2 anos. Esses acessos foram recolhidos através dos registos eletrónicos de todos os ginásios e health 


\section{Prática de exercício físico em adultos em contexto de ginásio}

clubs de forma individual. Posteriormente, dividimos os acessos em quatro momentos: 1) primeiros 6 meses (T1); 2) $6^{\circ}$ até ao $12^{\circ}$ mês (T2); 2) $12^{\circ}$ até ao $18^{\circ}$ mês (T3); e 4) últimos 6 meses (T4). Para análise, cada período compreendeu 26 semanas em que a frequência foi somada para um valor total. Usamos o valor de referência dos 6 meses de acordo com os pressupostos de estudos anteriores: a) a curva do abandono estabiliza a partir dos 6 meses (7); b) aproximadamente $50 \%$ dos praticantes abandonam a prática nos primeiros 6 meses (Buckworth \& Dishman, 2002; Radel et al., 2017); e c) de acordo com a fase da manutenção do modelo Transteórico (Prochaska \& DiClemente, 2005), o indivíduo faz o esforço de manter o comportamento após a sua realização durante 6 meses.

\section{Análise Estatística}

Todas as análises estatísticas foram realizadas com recurso ao programa Mplus 7.4 (Múthen \& Múthen, 2010). Como análise preliminar, foram calculadas as estatísticas descritivas (e.g., médias, desvio padrão, assimetria e curtose) bem como as correlações bivariadas. Dado as entradas nos ginásios e health clubs terem sido recolhidas como variáveis observáveis e considerando ser de interesse para este estudo os efeitos diretos e indiretos, seguimos as linhas orientadoras de modelos de equações estruturais, saturando o modelo definido como path analysis tal como proposto por Hair e colaboradores (2019). Nesse sentido, os índices tradicionais e incrementais não foram considerados dado o modelo proposto ser pré-especificado e totalmente saturado, exibindo um ajuste perfeito (Byrne, 2016). Para uma maior compreensão dos efeitos diretos e indiretos entre os quatro momentos, realizou-se um modelo de mediação em série. O modelo foi estimado de forma a obter o impacto dos diferentes períodos nos momentos seguintes, considerando T2 e T3 como mediadores entre T1 e T4. Mediação total será espectável caso o coeficiente dos efeitos indiretos totais seja superior e significativo face ao efeito direto entre T1 e T4 (Hayes, 2017). Os efeitos diretos, indiretos e totais foram analisados de acordo com as recomendações de Williams e Mackinnon (2008), reportando os coeficientes estandardizados e o Intervalo de Confiança (IC) a 95\%, sendo a estimativa considerada significativa caso o IC não englobe o valor de 0 .
Relativamente à taxa de abandono: 39\% ( $\mathrm{n}=$ 1867) abandonaram a prática de exercício físico entre $\mathrm{T} 1$ e T2, 25\% $(\mathrm{n}=1197)$ abandonaram a prática entre $\mathrm{T} 2$ e T3 e $10 \%(\mathrm{n}=479)$ abandonaram a prática entre T3 e T4. A média das entradas semanais apresenta um valor de aproximadamente duas sessões de treino por semana, em que o T4 apresenta a média mais elevada $(\mathrm{M}=2.31 ; \mathrm{DP}=.92)$. A Tabela 1 evidencia os valores de uma distribuição normal da frequência no ginásio durante os quatro momentos, dado os valores de assimetria e curtose estarem contidos dentro dos limites pré-estabelecidos. Todas as correlações são significativas entre os momentos analisados, sendo que a associação entre T3 e T4 apresenta o valor mais elevado.

De acordo com os resultados do modelo de equações estruturais (Figura 1), todos os efeitos diretos são positivos e significativos (ver Tabela 2), sendo que o efeito entre o período T3 para o período T4 apresenta a predição mais significativa. Igualmente, os coeficientes dos efeitos indiretos entre o período $\mathrm{T} 1$ para o período $\mathrm{T} 4$ e o período $\mathrm{T} 2$ para o período T4 são positivamente significativos. Nesse sentido testamos um modelo de mediação traçando regressões entre todos os momentos em análise. De acordo com os resultados do modelo de mediação (Figura 2), é possível de observar que o efeito direto é significativo. Igualmente, os efeitos indiretos são significativos entre todos os momentos (ver Tabela 2). Os resultados mostram a existência de mediação total entre o período $\mathrm{T} 1$ para o período $\mathrm{T} 4$, dado o efeito indireto total apresentar um coeficiente $(\beta=.91[.82$, 1.02]) superior e significativo comparativamente ao efeito direto entre o período T1 para o período T4. Nesse sentido, podemos assumir que o período T2 e T3 são mediadores entre o período T1 para o período T4.

\section{RESULTADOS}


Rodrigues, F., Macedo, R., Teixeira, D., Cid, L., \& Monteiro, D.

Tabela 1. Análise descritiva e correlações

\begin{tabular}{llcccccccc}
\hline & Período & $\mathrm{M}$ & $\mathrm{DP}$ & $\mathrm{S}$ & $\mathrm{K}$ & $\mathrm{T} 1$ & $\mathrm{~T} 2$ & $\mathrm{~T} 3$ & $\mathrm{~T} 4$ \\
\hline T1 & 1-6 meses & 60.34 & 7.42 & -1.43 & 2.12 & 1 & & & \\
T2 & 7-12 meses & 59.46 & 6.59 & -1.35 & 2.07 & .86 & 1 & & \\
T3 & $13-18$ meses & 62.44 & 4.44 & -1.18 & 1.62 & .79 & .86 & 1 & \\
T4 & $19-24$ meses & 63.51 & 4.02 & -1.24 & 1.74 & .82 & .90 & .94 & 1 \\
\hline
\end{tabular}

Notas: $\mathrm{M}=$ Média; $\mathrm{DP}=$ Desvio Padrão; $\mathrm{S}=$ Assimetria; $\mathrm{K}=$ Curtose; todas as correlações são significativas a $p \leq .001$.

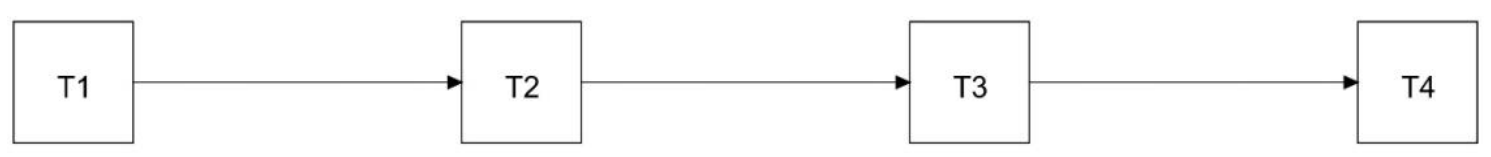

Figura 1. Modelo de equações estruturais

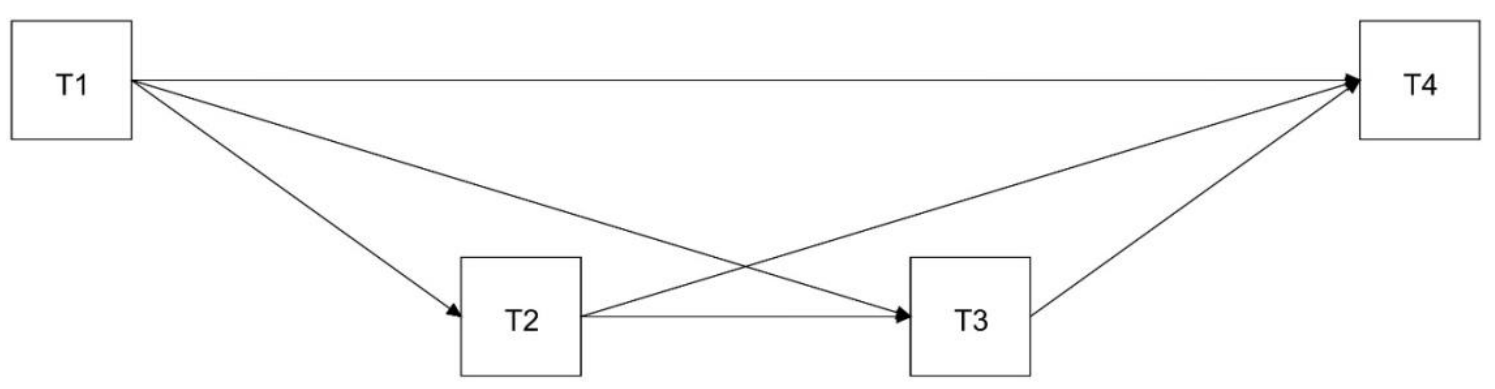

Figura 2. Modelo de mediação

Tabela 2. Efeitos diretos e indiretos do modelo de equações estruturais e de mediação

\begin{tabular}{|c|c|c|c|c|c|c|c|c|c|c|c|c|}
\hline & \multicolumn{6}{|c|}{ Modelo de Equações Estruturais } & \multicolumn{6}{|c|}{ Modelo de Mediação } \\
\hline & \multicolumn{3}{|c|}{ Diretos } & \multicolumn{3}{|c|}{ Indiretos } & \multicolumn{3}{|c|}{ Diretos } & \multicolumn{3}{|c|}{ Indiretos } \\
\hline & \multirow{2}{*}{$\beta$} & \multicolumn{2}{|c|}{ IC95\% } & \multirow{2}{*}{$\beta$} & \multicolumn{2}{|c|}{ IC95\% } & \multirow{2}{*}{$\beta$} & \multicolumn{2}{|c|}{ IC95\% } & \multirow{2}{*}{$\beta$} & \multicolumn{2}{|c|}{ IC95\% } \\
\hline & & LI & LS & & LI & $\mathrm{LS}$ & & LI & $\mathrm{LS}$ & & LI & LS \\
\hline $\mathrm{T} 1 \rightarrow \mathrm{T} 2$ & .85 & .76 & .95 & - & - & - & - & - & - & .88 & .81 & .94 \\
\hline $\mathrm{T} 1 \rightarrow \mathrm{T} 3$ & - & - & - & .74 & .63 & .83 & - & - & - & .23 & .04 & .41 \\
\hline $\mathrm{T} 1 \rightarrow \mathrm{T} 4$ & - & - & - & .70 & .61 & .80 & -.06 & -.15 & .04 & - & - & - \\
\hline $\mathrm{T} 2 \rightarrow \mathrm{T} 3$ & .86 & .80 & .92 & - & - & - & - & - & - & .65 & .48 & .83 \\
\hline $\mathrm{T} 2 \rightarrow \mathrm{T} 4$ & - & - & - & .82 & .75 & .88 & - & - & - & .51 & .37 & .65 \\
\hline $\mathrm{T} 3 \rightarrow \mathrm{T} 4$ & .95 & .92 & .98 & - & - & - & - & - & - & .58 & .45 & .72 \\
\hline
\end{tabular}

Notas: $\beta=$ coeficientes estandardizados; IC95\% = Intervalo de Confiança a 95\%; LI = Limite Inferior; LS $=$ Limite Superior. 


\section{Prática de exercício físico em adultos em contexto de ginásio}

\section{DISCUSSÃO}

Este estudo analisou o impacto do comportamento de praticantes de exercício físico em ginásios e health clubs em momentos consequentes ao longo de 2 anos. O modelo de mediação em série demonstra que o valor preditivo entre o período $\mathrm{T} 1$ para o período T2 é o mais forte e que o período T2 e o período T3 são mediadores entre os primeiros 6 meses de prática e os últimos 6 meses. Os resultados corroboram com os pressupostos do valor significativo do comportamento passado no comportamento futuro e serão discutidos com base na literatura disponível.

Os resultados realçam que a frequência de exercício físico em ginásio nos primeiros 6 meses constitui-se como um momento crucial para os 6 meses seguintes. De facto, sensivelmente $40 \%$ dos novos praticantes de ginásio $\mathrm{e}$ health clubs abandonaram a prática durante este período. Estes dados corroboram com estudos anteriores (Buckworth \& Dishman, 2002; Prochaska \& DiClemente, 2005; Radel et al., 2017), onde se explica que a taxa de abandono de um comportamento nos primeiros 6 meses é aproximadamente de $50 \%$. Ultrapassando a barreira dos primeiros 6 meses de prática, o valor preditivo mantém-se significativo, mostrando que o comportamento atual (i.e., T3) é explicado pelo "comportamento passado" T2 e indiretamente por T1. Isto reforça as evidências de Gardner (2015) e de estudos no contexto do exercício físico (Rodrigues et al., 2020), mostrando que o praticante de exercício está menos consciente do comportamento, dado este já fazer parte da sua rotina quotidiana. Além disso, tal como descrito por Prochaska e Diclemente (2005), o praticante já ultrapassou o período crítico dos primeiros 6 meses, estando mais predisposto a fazer o esforço de continuar a realizar o comportamento no futuro. De acordo com Sommers (2011) indivíduos que repetem o comportamento com maior frequência estão mais predispostos a repetirem o mesmo comportamento no futuro, ou seja, os resultados suportam a afirmação "diz-me o que fizeste no passado e dir-te-ei o que farás no futuro".

Interessantemente, no modelo de equações estruturais, T2 e T3 têm um efeito significativo indireto e direto respetivamente, com o período T4. Não obstante, de acordo com o modelo de mediação, $\mathrm{T} 1$ tem o efeito indireto significativo com $\mathrm{T} 4$ mediado por T2 e T3, mas não tem um efeito direto significativo. Estes resultados reforçam a ideia de que, os primeiros 6 meses constituem-se como sendo um período de adaptação e mudança comportamental, tal como descrito pela fase da ação do modelo Transteórico (Prochaska \& DiClemente, 2005). Isto é, o indivíduo está a realizar o comportamento de forma a que se torne habitual, não estando totalmente interiorizado como um comportamento rotineiro. $\mathrm{O}$ praticante tem a intenção de mudar, passando de um comportamento fisicamente inativo para a prática regular de exercício, no entanto, não garante que a mudança se mantenha ao longo do tempo. Nesse sentido, a frequência regular sem episódios de desistência no T2 e T3 são fundamentais para que o praticante de exercício físico interiorize o comportamento como sendo parte deste.

De acordo com estes resultados, os instrutores de fitness devem focar-se na promoção de um conjunto de atividades moderadamente exigentes e desafiantes, que possibilitem o desenvolvimento das capacidades técnicas do praticante, de forma a satisfazer as necessidades psicológicas básicas, diretamente relacionadas com a motivação mais autodeterminada (Rodrigues et al., 2020b). Paralelamente, os instrutores de fitness devem igualmente incentivar o envolvimento ativo dos praticantes no seu progresso, permitindo-lhes escolher quais os exercícios a realizar, dando-lhes liberdade para propor novos desafios e incutindo-lhes autonomia para serem responsáveis pelo próprio progresso, principalmente neste período crítico (T2 e T3), para que os praticantes possam continuar a internalizar o comportamento como fazendo parte integrante do seu quotidiano.

Os resultados mostram que a taxa de abandono foi de $39 \%$, entre $\mathrm{T} 1$ e T2; $25 \%$ entre T2 e $\mathrm{T} 3$, e $10 \%$ entre $\mathrm{T} 3$ e $\mathrm{T} 4$. A percentagem de desistências nos primeiros 6 meses está ligeiramente abaixo das descritas por vários autores (Radel et al., 2017). Não obstante, abandonaram no total 3543 praticantes de exercício físico ao longo de 2 anos sem retorno à prática, confirmando a tendência atual da elevada taxa de abandono (AGAP, 2018), principalmente nos primeiros meses, diminuindo a taxa de abandono ao longo do tempo (Sperandei et al., 2016). Os números de abandono podem estar relacionados com diversos indicadores sociodemográficos (e.g., mudança de zona de 


\section{Rodrigues, F., Macedo, R., Teixeira, D., Cid, L., \& Monteiro, D.}

residência) e temporais (e.g., mudança da estação). Nesse sentido não se pode inferir que as pessoas tenham desistido da prática de exercício físico, apenas que tenham abandonado a sua prática em contexto de ginásio e health club. Futuros estudos deverão avaliar a continua prática (ou total desistência) de exercício físico em espaços verdes ou de outra forma regular, tais como atividades comunitárias.

A frequência rondou as 2 vezes por semana, aumentando ligeiramente ao longo dos quatro momentos. Estes resultados são semelhantes aos obtidos por Hooker (2016), mostrando que a frequência de 2 vezes por semana, parece ser o estímulo mínimo para que o comportamento se possa tornar habitual. Acresce ainda o facto de que quem praticou exercício físico sem episódios de desistência manteve-se mais tempo na prática. De acordo com Lally e colaboradores (2011) não basta apenas realizar o comportamento, mas sim ser consistente e repeti-lo para reforçar a sua automaticidade. Caso o praticante se tenha comprometido a frequentar o ginásio/health clube pelo menos duas vezes por semana, sem interrupção nos primeiros 6 meses, a probabilidade de continuar a praticar exercício físico nos meses seguintes é maior.

\section{Limitações}

Apesar de esta investigação apresentar avanços científicos relevantes para o contexto em questão, é fundamental perceber as limitações existentes. $\mathrm{O}$ desenho prospetivo com um acompanhamento do comportamento ao longo de dois anos limita a interpretação de inferência, sugerindo que se devem criar intervenções que possam reduzir o número de pessoas que abandonaram a sua prática ao longo dos dois anos. A literatura existente tem comprovado que o recurso a técnicas de mudança comportamental pode levar a uma melhoria na comunicação do instrutor de fitness, facilitando a relação praticante-instrutor e assim aumentar a adesão à prática de exercício físico (Ntoumanis et al., 2017).

Por último, só foi possível recolher dados de alguns ginásios e health clubs em Portugal, sendo que os dados não poderão ser generalizados para toda a operacionalização do fitness a nível nacional. Acrescido, não se analisou com detalhe a possibilidade de haver um efeito moderador de algumas variáveis sociodemográficas (e.g., idade, sexo), bem como na escolha das atividades de fitness (e.g., musculação vs. aulas de grupo). Nesse sentido, este estudo abre portas para mais estudos que possam examinar o fenómeno da prática de exercício físico, bem como a compreensão das causas e consequências do comportamento ao longo do tempo.

\section{CONCLUSÃO}

Cabe aos gestores de ginásios/health clubs estarem em constante processo de alerta do comportamento dos novos praticantes de forma a conseguirem traçar o desfecho (i.e., abandono ou persistência) num período de 2 anos. Tal como reportado neste estudo, os praticantes que frequentaram o ginásio/health club pelo menos duas vezes por semana sem interrupção foram aqueles que mais tempo tiveram na prática e/ou que continuaram após os 2 anos de acompanhamento. Pelo contrário, praticantes com episódios de interrupção/com uma frequência inferior a 2 vezes por semana desistiram do comportamento durante o período em análise. Isto demonstra que indivíduos que falham a oportunidade de irem ao ginásio poderão estar em risco de abandonar o comportamento.

Identificar as variáveis determinantes para a formação do comportamento padrão torna-se crucial. Variáveis como os níveis de motivação poderão determinar a realização de exercício físico no futuro (Rodrigues et al., 2020b). Cada praticante de exercício físico em ginásio ou health club deve ser visto como um todo, pelo qual deve ser acompanhado constantemente de forma individual, procurando satisfazer as necessidades deste. Os dados aconselham uma atenção nos novos praticantes, especialmente aqueles com menos de 6 meses de experiência em exercício físico, dado estarem mais suscetíveis a abandonarem a prática de exercício físico. A análise da frequência semanal constitui-se como indicador a ser tido em consideração pelos gestores de ginásios e health clubs aquando do acompanhamento por parte dos técnicos profissionais. Uma "falta" por parte do praticante sem aviso pode constituir-se como um sinal de alerta.

Concluindo, após apresentação, análise e discussão dos resultados, é importante refletir sobre a importância do presente trabalho. A análise da frequência dos praticantes de exercício físico em ginásio e health club irá permitir, por exemplo aos 


\section{Prática de exercício físico em adultos em contexto de ginásio}

gestores e diretores técnicos de um operador fitness, ter indicações acerca do trabalho desenvolvido pelos instrutores de fitness e intervir de forma a melhorar os indicadores de qualidade como forma de melhorar as componentes técnicas destes profissionais na retenção dos utilizadores (Teixeira et al., 2020); ou aos instrutores, retificar e/ou orientar a sua intervenção profissional de acordo com a perceção dos seus praticantes, adequando a prescrição de acordo com as necessidades dos mesmos, dado os comportamentos interpessoais por parte dos instrutores desempenharem um papel crucial (Rodrigues et al., 2019c). Estes exemplos remetem-nos para o problema inicialmente apresentado, focado na necessidade de os ginásios implementarem estratégias para diminuição do abandono e/ou aumento da prática de exercício físico regular a longo prazo (Rodrigues et al., 2020b). Sabendo que os comportamentos interpessoais entre os praticantes e os instrutores antecede a intenção e que, a esta, está associada a persistência (Rodrigues et al., 2020b), esta análise do comportamento poderá ser um indicador útil de forma a melhorar a qualidade do serviço prestado pelos instrutores no âmbito das atividades de grupo de fitness.

\section{REFERÊNCIAS}

AGAP. Barómetro do Fitness em Portugal 2018 (2018). Universidade Autónoma de Lisboa: Lisboa.

Arem, H., Moore, S., Patel, A., Hartage, P., Gonzalez, A., Matthews, C. (2015) Leisure time physical activity and mortality: a detailed pooled analysis of the dose-response relationship. Journal of American Medical Association, 175(6), 959-967. https://doi.org/10.1001/jamainternmed.2015.053 $\underline{33}$

Barg, J., Ferguson, M. (2000). Beyond behaviorism: on the automaticity of higher mental processes. Psychological Bulletin, 126(6): 925-945. https://doi.org/10.1037/0033-2909.126.6.925

Brennder, P., DeLamater, J. (2016). Lies, damned lies, and survey self-reports? Identity as a cause of measurement bias. Social Psychology Quarterly, 79(4): 333-354. Doi: https://doi.org/10.1177/0190272516628298

Brickell, T., Chatzisarantis, N., Pretty, G. (2006). Using past behavior and spontaneous implementation intentions to enhance the utility of the theory of planned behavior in predicting exercise. British Journal of Health Psychology, 11 , 249-262. https://doi.org/10.1348/135910705X52471

Buckworth, J., Dishman, R. (2002). Exercise adherence. In Tenenbaum, G., \& Eklund, R. (eds.), Handbook of sport psychology (pp. 509536). Human Kinetics: United Kingdom. https://doi.org/10.1002/9781118270011.ch23

Byrne, B. (2016). Structural equation modelling with Mplus: Basic Concepts and Programming. Routledge: United Kingdom. ISBN: 9781848728394

Caspersen, C., Powell, K., Christenson, G. (1985). Physical activity, exercise, and physical fitness: definitions and distinctions for health-related research. Public Health Reports, 100(2), 126131.

Eurobarómetro. (2018). Eurobarometer 472 Report. European Commission.

Gardner, B. (2015). A review and analysis of the use of 'habit' in understanding, predicting and influencing health-related behavior. Health Psycholgy Review, 3:1-19. https://doi.org/10.1080/17437199.2013.876238

Gomes, R., Morais, R., Carneiro, L. (2017). Predictors of exercise practice: from intention to exercise behavior. International Journal of Sports Science, $\quad 7(2)$ : $\quad 56-65$. https://doi.org/10.5923/j.sports.2017072.06

Hagger, M. (2018). Habit and physical activity: theoretical advances, practical implications, and agenda for future research. Psychology in Sports and Exercise, 42:118-129. https://doi.org/10.1016/j.sychsport.2018.12.00 $\underline{7}$

Hagger, M., Chatzisarantis, N. (2014). An integrated behavior change model for physical activity. Exercise and Sports Science Review, 42(2): 6269.

https://doi.org/10.1249/JES.000000000000008

Hair, J., Black, W., Babin, B., Anderson, R. (2019). Multivariate data analysis (8th ed.). Cengage Learning. ISBN: 978147756545 


\section{Rodrigues, F., Macedo, R., Teixeira, D., Cid, L., \& Monteiro, D.}

Hayes, A. (2017). Introduction to mediation, moderation, and conditional process analysis. Guilford Press: New York. ISBN: 9781462534654

Hooker, S., Ross, K., Ranby, K., Masters, K., Peters, J., Hill, J. (2016). Identifying group at risk for 1-year membership termination from a fitness center at enrollment. Preventive Medicine Reports, 4: 563-568. https://doi.org/10.1016/j.pmedr.2016.10.016

Janssen, I., LeBlanc, A. (2010). Systematic review of the health benefits of physical activity and fitness. International Journal of Behavioral Nutrition and Physical Activity, 7:40. https://doi.org/10.1186/1479-5868-7-40

Lally, P., Wardle, J., Gardner, B. (2011). Experiences of habit formation: a qualitative study. Psychology, Health \& Medicine, 16(4): 484489.

https://doi.org/10.1080/13548506.2011.555777 $\underline{4}$

Liu, S., Eaton, C., Driban, J., McAlindon, T., Lapane, K. (2016). Comparisons of self-report mand objective measures of physical activity in US adults with osteoarthritis. Rheumatology International, 36(10): 1355-1364. https://doi.org/10.1007/s00296-016-3537-9

Múthen, B., Múthen, L. (2010). Mplus user's guide (6th ed.). CA Múthen \& Múthen: Los Angeles.

Nomura, T., Yoshimoto, Y., Akezai, Y., Sato, A. (2009). Changing behavioral patterns to promote physical activity with motivational signs. Environmental Health Preventive Medicine, $\quad 14(1)$ : 20-25. https://doi.org/10.1007/s12199-008-0053-X

Ntoumanis, N., Thogersen-Ntoumani, C., Quested, E., Hancox, J. (2017). The effects of training group exercise class instructors to adopt a motivationally adaptive communication style. Scandinavian Journal of Medicine and Science in Sports, 27(9), 1026-1034. https://doi.org/10.1111/sms.12713

OMS. (2018). Global action plan for physical activity. World Health Organization.

Prochaska, J., DiClemente, C. (2005). The transtheoretical approach. In Norcross, J. \&
Goldfield, M. (eds.), Exford series in clinical psychology. Handbook of psychotherapy integration (pp. 147-171). Oxford University Press: New York. https://doi.org/10.1093/med:psych/978019516 5791.003.0007

Rodrigues, F., Cid, L., Forte, P., Gonçalves, C., Machado, S., Neiva, H., Teixeira, D., Monteiro, D. (2020a). A perceção de divertimento em jovens, adultos e idosos: um estudo comparativo. Cuadernos De Psicología Del Deporte, 20(2), 26-36. Doi: $10.6018 / \mathrm{cpd} .403391$

Rodrigues, F., Teixeira, D., Neiva, H., Cid, L., Monteiro D. (2020b). Understanding Exercise Adherence: The Predictability of Past Experience and Motivational Determinants. Brain Science, 10(2):98. Doi:10.3390/brainsci10020098

Rodrigues, F., Teixeira, D., Cid, L., Monteiro, D. (2019a). Have you been exercising lately? Testing the role of past behavior on exercise adherence. Journal of Health Psychology. Doi:10.1177/1359105319878243

Rodrigues, F., Teixeira, D., Cid, L., Monteiro, D. (2019b). Promoting Physical Exercise participation: the role of interpersonal behaviors for practical implications. Journal of Functional Morphology and Kinesiology, 4,40. https://doi.org/10.3390/jfmk4020040

Rodrigues, F., Neiva, H., Marinho, D., Mendes, P., Teixeira, D., Cid, L., Monteiro, D. (2019c). Assessing need satisfaction and frustration in Portuguese exercise instructors: scale validity, reliability, and invariance between gender. Cuadernos de Psicología del Deporte, 19(1), 233-240. https://doi.org/10.6018/cpd.347331

Rodrigues, F., Bento, T., Cid, L., Neiva, H., Teixeira, D., Moutão, J., Marinho, D., \& Monteiro, D. (2018). Can interpersonal behavior influence the persistence and adherence to physical exercise practice in adults? A systematic review. Frontiers in Psychology, 9:2141. Doi: https://doi.org/10.3389/fpsyg.2018.02141

Radel, R., Pelletier, L., Pjevac, D., Cheval, B. (2017). The links between self-determined motivations and behavioral automaticity in a variety of real- 


\section{Prática de exercício físico em adultos em contexto de ginásio}

life behaviors. Motivation and Emotion, 41:443. https://doi.org/10.1007/s11031-017-9618-6

Sperandei, S., Vieira, M., Reis, A. (2016). Adherence to physical activity in an unsupervised setting: explanatory variables for high attrition rates among fitness center members. Journal of Science and Medicine in Sports, 19(11): 916920.

https://doi.org/10.1016/j.sams.2015.12.522

Sommers, L. (2011). The Theory of Planned Behavior and the impact of past behavior. International Business and Economics Research Journal, 10(1): 91-110. https://doi.org/10.19030/iber.v10i1.930.

Teixeira, D., Monteiro, D., Rodrigues, F., Sousa, M., Chaves, C., Cid, L. (2020). Ginásios e health clubs em Portugal: Estaremos perante uma república das bananas? Motricidade, 16(1), 317. https://doi.org/10.6063/motricidade. 19688

Verplanken, B. (2006). Beyond frequency: habit as a mental construct. British Journal of Social

\section{Agradecimentos}

Este estudo foi suportado pela Fundação para Ciência e Tecnologia com o número de registo UID04045/2020para os autores Luís Cid e Diogo Monteiro. Este estudo também foi suportado pela Fundação para a Ciência e a Tecnologia, I.P., Projeto $\mathrm{N}^{\circ}$ UIDP/04748/2020 para o autor Filipe Rodrigues

This research received an honorable mention by the COP/Millenium BCP Sport Sciences awards of 2019.

\section{Conflitos de interesse}

Nada a declarar.
Psychology, 45(3): https://doi.org/10.1348/014466605X49122

Williams, J., Mackinnon, D. (2008). Resampling and distribution of the product methods for testing indirect effects in complex models. Structural Equation Modelling, 15(1): 23-51. https://doi.org/10.1080/10705510701758166

Bay-Hinitz, A.K., Peterson, R.F., Quilitch, H.R. (1994). Cooperative games: A way to modify aggressive and cooperative behaviors in young children. Journal of Applied Behavior Analysis, 27(3), 435-446. 